\title{
Bibliographie de la Société de Notre-Dame de Montréal (1639-1663) et de ses membres, accompagnée de notes historiques et critiques (deuxième partie)
}

\section{Marie-Claire Daveluy}

Volume 5, numéro 4, mars 1952

URI : https://id.erudit.org/iderudit/802146ar

DOI : https://doi.org/10.7202/802146ar

Aller au sommaire du numéro

Éditeur(s)

Institut d'histoire de l'Amérique française

ISSN

0035-2357 (imprimé)

1492-1383 (numérique)

Découvrir la revue

Citer ce document

Daveluy, M.-C. (1952). Bibliographie de la Société de Notre-Dame de Montréal (1639-1663) et de ses membres, accompagnée de notes historiques et critiques (deuxième partie). Revue d'histoire de l'Amérique française, 5(4), 603-616. https://doi.org/10.7202/802146ar 


\section{BIBLIOGRAPHIE}

Bibliographie de la Société de Notre-Dame de Montréal (1639-1663)... accompagnée de notes historiques et critiques.

$$
\text { DEUXIÈME PARTIE }
$$

\section{BIO-BIBLIOGRAPHIE DES MEMBRES}

Note: Voici le plan que nous avons adopté:

I. - Bio-Bibliographie des associés de Montréal pris collectivement ou mentionnés, isolément, les uns ou les autres, dans un seul ouvrage.

II. - Bio-Bibliographie des associés de Montréal, rangés d'après leur ordre d'entrée dans la Société de NotreDame. Sous ordre alphabétique pour certaines années.

III. - Index général.

N.B. Les bio-bibliographies n'accusent aucun caractère exhaustif; elles restent sélectives dans la présentation des œuvres écrites par ou sur les associés de Montréal.

Bibliographie des associés de Montréal, pris collectivement, ou mentionnés isolément, les uns ou les autres, dans un seul ouvrage.

Ouvrages généraux, biographies, études d'ensemble et de détail, lettres, mémoires... - Classement chronologique (d'après l'ordre des manuscrits).
A. - Le 17e siècle
B. - Le 18e siècle
C. - Le $19 \mathrm{e}$ siècle
D. - Le 20e siècle 


\section{A. - LE 17e SIÈCLE}

57. - État de la Maison du roi Louis XIII; de celle de sa mère, Marie de Médicis, de ses sœurs Chrestienne, Elisabeth et Henriette de France, de son frère Gaston d'Orleans; de sa femme, Anne d'Autriche... comprenant les années 1601 à 1665. Publié par Eugène Griselle,... Paris, Éditions de documents d'histoire, Paul Cantin, administrateur, 13, rue Lacépède, 1912. VIII - 412 pages. $22 \times 14 \mathrm{~cm}$.

N.B. Les notes, les commentaires, et la table alphabétique qui est considérable, forment l'armature des documents présentés et nous permettent de mieux utiliser les recherches de M. Griselle. La Société de Notre-Dame de Montreal, nous le savons, comptait dans ses rangs des personnes illustres et influentes à la Cour. Celles-ci émargent donc avec leurs titres et leurs fonctions, au budget des maisons royales et princières. Entre autres: le prince de Conti, le duc de Ventadour, Henry-Louis Habert de Montmort, Bertrand Drouart de Sommelan, la princesse de Condé (Charlotte de Montmorency), Madame Isabelle Blondeau de Villesavin, Madame Séguin... II faut aussi consulter du même M. Griselle: Supplément à la Maison du roi Louis XIII (Paris, 1912), et Maisons de la Grande Mademoiselle et de son père, Gaston d'Orléans... (ibid.)

58. - Saint Vincent de Paul, 1576-1660. - Correspondance, Entretiens, Documents... [ 1607-1660]... Édition publiée et annotée par Pierre Coste, prêtre de la Mission. Paris, Librairie Lecoffre. - J. Gabalda, éditeur, 90, rue Bonaparte, 19201925. 14 volumes. $23.5 \times 14.5 \mathrm{~cm}$.

N.B. L'ouvrage d'un érudit de qualité tel que l'abbé Pierre Coste ne laisse vraiment rien à désirer. Deux tables: 1 . Table des correspondants de saint Vincent de Paul; 2. Table bibliographique apparaissant au tome VIII (le dernier de la Correspondance). Le vol. XIV est entièrement consacré à une table générale qui couvre 648 pages. Signalons, en outre, la présence de notes et de références, toujours substantielles, dans presque chaque page des 13 volumes, comptant, environ, 600 pages chacun. L'information est abondante et exacte; les personnages, bien identifiés. Parmi les correspondants de saint Vincent de Paul, nous relevons les noms de six associés de Montréal: les frères Brandon du Laurent et Brandon de Bassancourt, le prince de Conti, Madame de Liancourt, duchesse de la RocheGuyon (née Jeanne de Schomberg), M. Jean-Jacques Olier, la baronne Gaston de Renty, née Elisabeth de Balsac d'Entragues). Dans la table générale du tome XIV, vingt-quatre Messieurs et dames de Montréal apparaissent. C'est dire l'importance de cet ouvrage qui nous fournit des renseignements que l'on ne trouve nulle part ailleurs. Nous y apprenons, par exemple, la date d'ordination de M. Laisné de la Marguerie, certains 
détails sur la mort du Comte de Chaudebonne, le nom de l'évêché proposé en 1635 à M. Olier, etc...

Dans l'Introduction que signait M. François Verdier, supérieur général de la Congrégation de la Mission, en juillet 1931, pour présenter la Vie de saint Vincent de Paul, du même auteur (Pierre Coste), le distingué religieux déclarait: "Dirai-je que votre Vie de saint Vincent, [ est le] couronnement de votre grandiose monument à votre saint..." Comment ne pas accepter le redoutable qualificatif: grandiose, quand on songe à l'ampleur des travaux, et à la science historique rigoureuse de l'abbé Coste?

59. - Annales de la Compagnie du St-Sacrement, 1627-1666, par le Comte René de Voyer d'Argenson [ 1623-1700 ] publiées et annotées par le R.P. Dom H[enri] Beauchet-Filleau, moine bénédictin. Marseille, Typographie \& Lithographie SaintLéon, rue des Princes, 78, 1900. XIV - 319 pages. 25 x 14.5cm.

N.B. Nous savons que le premier projet de fondation de la Compagnie date de 1627 et qu'elle fut organisée en 1630. Interdite en 1660, son "total anéantissement" ne vint qu'en 1666, lors de l'établissement du Séminaire des Missions étrangères "le dernier ouvrage de la Compagnie", aujourd'hui encore vivant et agissant sur les plages les plus lointaines. Le Comte René II de Voyer d'Argenson se décida d'écrire les Annales ci-dessus en 1694, dans l'espoir de voir renaître la société. Il eut accès aux registres (commencés en juillet 1630) et en tira des textes substantiels. Il les divisa en deux parties (18 chapitres, de 1630 à 1656 pour la première; et 14 chapitres, de 1657 à 1665 pour la seconde). Un appendice clôt le livre manuscrit. C'est le Père Le Lasseur, un savant jésuite, qui le découvrit vers 1865, presque deux siècles après qu'il eut été écrit. Il fit part de sa trouvaille au bénédictin Dom Beauchet-Filleau, qui l'édita avec d'érudits commentaires et de nombreuses notes et références, longtemps après la mort du Père Le Lasseur, et douze ans après les copieux et agréables articles du Père Charles Clair, s.j., sur le sujet. (Les Études, Paris, nov. et déc. 1888, et janv. et fév. 1889, tomes 45 et 46 de la collection). Nous ne pouvons laisser dans l'ombre, l'action bienfaisante, - sinon toujours assez tolérante, disent beaucoup d'historiens, - de cette Compagnie à laquelle appartiennent les principaux membres de la Société de Montréal. Quelques-uns, comme le baron de Renty, Élie Laisné de la Marguerie, Antoine Barillon de Morangis, Jean-Antoine de Mesmes d'Irval, Christophe Du Plessis de Montbard, Jean de Garibal, Philibert Brandon du Laurent, Bertrand Drouart de Sommelan, le duc, puis chanoine Henry de Lévis de Ventadour, y tinrent un rôle prépondérant, soit en qualité de fondateur, de supérieurs, de directeurs ou de secrétaire. Nous pouvons relever, en tout cas, les noms de 21 associés de Montréal ayant fait partie de la Compagnie du SaintSacrement.

Mais de là à faire de la Société de Notre-Dame de Montréal, un Comité de la célèbre Compagnie, et lui attribuer l'œuvre colonisatrice de Montréal, 
il y a loin, malgré ce qu'en croit là-dessus, Raoul Allier, l'auteur de la Cabale des dévots (Paris, 1902) et après lui, l'école historique protestanto de chez nous. Qu'on se soit servi de la Compagnie, alors que MM. de la Dauversière, de Fancamp et Olier, fondaient la Société de Montréal, nous voulons bien l'admettre. Du reste, c'était agir conformément à ses règlements: "Quand Dieu inspirera à quelqu'un de se servir de la Compagnie pour exécuter ses pieuses intentions... il pourra prendre et nommer à cet effet deux ou trois de ces messieurs comme particuliers, qui seront néanmoins approuvés et avoués par la Compagnie, lesquels s'en acquitteront diligemment". Et puis, le principe fondamental du secret, qui a été observé avant même qu'on eût donné un nom à la Compagnie, fin de 1630, comment se serait-il accommodé d'une entreprise comme celle de la fondation et de la colonisation d'une ville? Et la présence des femmes, agissant, collaborant avec les hommes dans la Société de Montréal, ce que nul groupement ne tolérait dans la Compagnie, où même on ne voulut jamais de "Compagnie de Dames". Enfin, il faudrait écarter le rôle prépondérant, personnel, providentiel, tenu par M. de La Dauversière dans la fondation de notre ville. Nous ne pouvons pourtant rejeter des faits historiques dûment prouvés. La filiale de la Compagnie établie à La Flèche, en 1635, le fût certes par les soins des frères Le Royer, René et Jérôme, qui centralisaient autour d'eux, les œuvres de charité et de dévotion; mais les projets personnels de Jérome, ce mystique inspiré, demeurent antérieurs à l'installation d'une filiale, puisque déjâ, plusieurs personnes connaissaient ces projets et même les rejetaient comme "de pieuses chimères".

60. - La Compagnie du T.-S.-Sacrement de l'Autel à Marseille. Documents [1639-1702 ], publiés par Raoul Allier. Paris, Librairie Honore Champion, éditeur, 5 quai Malaquais, 1909. XIX - 492 pages. $23.5 \times 15 \mathrm{~cm}$.

N.B. En tête du titre, on lit: Une société secrète au XVIIe siècle. II y a dans cette édition des papiers de la Compagnie de précieuses communications adressées par la Compagnie de Paris à sa filiale de Marseille. Une. lettre de 1646 annonce la mort de l'abbé Thomas Le Gauffre, cet évêque nommé du Canada. On nous révèle au sujet du legs testamentaire de ce dernier à la Compagnie, qui est nommée, en outre, exécutrice des dernières volontés du saint prêtre, la décision prise par la Compagnie de n'accepter ni l'un ni l'autre, à cause du secret plus que jamais exigé en toutes les occasions. Une autre lettre expédiée à Marseille fait part du décès du baron Gaston de Renty, alors supérieur de la Compagnie de Paris, pour la onzième. fois. Ces deux pièces seules ne nous justifient-elles pas de signaler tout particulièrement l'excellent ouvrage de Raoul Allier? Nous joindrons ici, du même auteur, l'Histoire de la Compagnie du T.-S.-Sacrement à Toulouse (Paris, 1914) qui contient en appendice des pièces justificatives intéressantes, où l'on voit de nouveau l'action exercée par Gaston de Renty auprès de groupes sociaux les plus divers; il s'agit ici de gens de métiers, de serviteurs- 
cordonniers toulousains. Quant à l'œuvre aujourd'hui célèbre de Raoul Allier, que nous mentionnions tout à l'heure, la Cabale des dévots, nous en reparlerons au long, en inscrivant les ouvrages du XXe siècle.

61. - La Compagnie secrète du Saint-Sacrement. Lettre du groupe parisien au groupe marseillais (1639-1662) publiés par Alfred Rebelliau. Paris, Librairie Honoré Champion, éditeur, 5, quai Malaquais, 1908. $17 \times 13 \mathrm{~cm}$.

N.B. M. Allier dans l'ouvrage précédent (no 60), nous déclare: "Quiconque voudra se servir de la présente publication [ la sienne] devra avoir constamment sous la main, l'excellent recueil de pièces que M. Alfred Rebelliau nous a récemment donné... Nos deux livres sont inséparables..." Nous avons lu et relu les deux ouvrages, observant avec M. Allier que "les documents s'enchevêtrent les uns dans les autres." Quel dommage que ces œuvres soient devenus aujourd'hui rarissimes, presque introuvables.

63. - Jean Loret. La Muze historique, ou recueil de lettres en vers contenant les nouvelles du temps écrites à son Altesse Mademoizelle de Longueville, depuis duchesse de Nemours: 1650-1665, par J. Loret. Nouvelle édition revue sur les Manuscrits et les éditions originales, et augmentée d'une introduction de notes et d'une table générale des matières par MM. J. Ravenel et Ed. V. de la Pelouze [ et de Charles Livet ]. Paris, Jannet, 1857-1878. 4 vol. $24 \mathrm{~cm}$.

N.B. Même s'il les qualifie de vers burlesques, avec la plupart des critiques, l'historien, placé en face de cette ouvre, constate qu'on y fait grâce d'aucun détail, soit quand il s'agit des somptuosités de table des Liancourt et des Séguier, soit quand on y relate, non sans une emphase regrettable, la mort des plus saints personnages. Il y a énormément à retenir dans les récits de Jean Loret, gazettier attentif, et courtisan parfait.

Plusieurs des Messieurs et Dames de Montréal apparaissent dans ces pages fastidieuses: Les Liancour, Mme Séguin, Habert de Montmort et sa famille, la princesse de Montmorency, Madame de Villesavin, Laisné de la Marguerie, le saint M. Olier, Louis Seguier de Saint-Firmin, les Barillon de Morangis et d'Ammoncourt, l'oncle et le neveu, la Chancelière Séguier... d'autres encore. Anecdotes, scènes typiques, événements les plus divers sont racontés au jour le jour, et permettent de connaître beaucoup de faits intimes à côté de ceux qui étaient déjà de notoriété publique.

64. - Gédéon Tallemant des Réaux, 1619-1692. - Historiettes... [ Rédigées de 1657 à 1659 ]. Troisième édition par MM. de Monmerqué et Paulin Pâris. Paris, Techener, 18541860. 9 volumes, $23 \times 15 \mathrm{~cm}$.

N.B. Voici, sur ce mémorialiste, le jugement de MM. Émile Bourgeois et Louis André (Les Sources de l'Histoire de France (1610-1715). II. Mémoi- 
res et Lettres. Paris, Picard, 1913). "Si l'on met à part les exagérations provoquées par la malignité et l'amour de la médisance, cette œuvre, toute impersonnelle, que l'auteur n'a rédigée ni dans un but de justification, ni pour se faire valoir, dans laquelle il ne parle pas de lui-même, est unique au XVIIe siècle: ces "mémoires des autres", comme Tallemant a spirituellement appelé ses Historiettes, ont pour l'étude des mœurs et des idées une importance indéniable." MM. Bourgeois et André se réclament ailleurs de l'opinion de Sainte-Beuve: "Il ne faut pas traiter Tallemant à la légère, a dit le fameux critique, ni le contredire sans preuve." On peut aussi faire appel... aux travaux savants et si agréables, d'Émile Magne: La Joyeuse Jeunesse de Tallemant des Réaux et la Fin troublée de Tallemant des Réaux, 2 vol., (Paris, Émile-Paul, 1921-1922). Parent de Madame de Rambouillet, Tallemant fréquenta son salon, tout comme ceux "de la bourgeoisie financière, commerçante et opulente". Plusieurs associés de Montréal n'échappèrent pas à ses traits railleurs, tels: Antoine Barillon de Morangis, Madame de Bullion, M. de Chaudebonne, la princesse de Condé, la Chancelière Séguier, M. des Mesmes d'Irval, les Liancourt, Madame de Villesavin, Louis Séguier de Saint-Firmin, M. de Bretonvilliers et sa famille, le prince de Conti... Si quelques portraits sont de véritables charges et ne nous impressionnent guère puisque nous pouvons rétablir les faits dans leur intégrité historique, d'autres reconstituent autour des personnages des cadres que nous connaissions plus ou moins. Ils nous aident à expliquer l'humeur et les gestes des puissants, ou des petites gens, vivant au grand siècle.

65. - Louis Moreri [ 1643-1680], prêtre, docteur en théologie. - Grand dictionnaire historique... Nouvelle édition, revue, corrigée et augmentée par M. Drouet. Paris, 1759. 10 vol. Grand in-folio.

N.B. Lorsque les travaux biographiques ne sont pas nombreux ou difficiles d'accès, autour des personnages à l'étude, l'historien doit fouiller tous les ouvrages de référence dont l'autorité est reconnue. Moreri ne se consulte pas en vain. C'est un érudit qui a su se renseigner sur les célébrités du siècle où il a vécu, le 17e. De petits détails s'ajoutent souvent en le consultant; et même, parfois, il est le seul à dresser des notices biographiques sur des figures de second plan au point de vue de l'histoire universelle, mais de premier plan lorsqu'il s'agit d'histoires nationales ou locales. Nous y retrouvons plusieurs associês de Montréal; et aussi ceux qui les aidèrent à accomplir leur mission, tel ce provincial franciscain de Paris, le Père Charles Rapine, récollet, qui facilitait à Jeanne Mance des entrevues fructueuses avec Madame de Bullion.

Rappel: voir aussi sur le 17 e siècle, les nos $45,46,48,49,51,54$, de cette bibliographie (le partie). 


\section{B. - LE 18e SIẼCLE}

66. - Joseph Grandet, p.s.s., 1646-1724. - Les Saints Prêtres français du XVIIIe siècle. Ouvrage publié pour la première fois, d'après le manuscrit original, par G. Letourneau, p.s.s. supérieur du Séminaire d'Angers. Angers, Germain et G. Grassin; Paris, A. Roger \& F. Chernoviz, 1897. 3 volumes, $25.5 \times 17 \mathrm{~cm}$.

N.B. Les deux manuscrits de l'ouvrage, dont les rédactions sont authentiques, appartiennent: le premier, écrit avant 17\%0, au Séminaire d'Angers; le second, écrit en 1720, au Séminaire de Saint-Sulpice, à Paris. M. Letourneau a utilisé les deux manuscrits dans son édition. "L'un, nous explique-t-il, contenait des annotations et des corrections nombreuses faites de la main de l'auteur; l'autre, présentait des notices que ne donnait point le premier manuscrit, et fournissait, en outre, un récit plus parfait des notices parallèles." Grâce à l'ouvrage de M. Grandet, aux notes et aux références de son éditeur, nous possédons sur quelques prêtres de la Société de Notre-Dame de Montréal, de courtes biographies, laissant voir les lignes essentielles des modèles. Notons celles des abbés Kériolet, Le Gauffre, Olier et Bretonvilliers. Des souvenirs sur cinq autres associés de Montréal prennent place dans les diverses notices des volumes I et II.

67. - François-Xavier de Charlevoix, s.j. [1682-1761 ], Histoire et description générale de la Nouvelle-France... A Paris, chez Pierre-François Giffart, rue Saint-Jacques, à Sainte-Thérèse, M. DCC. XLIV. 3 volumes. Cartes et figures. In-4.

N.B. Le Père de Charlevoix fut certainement le premier écrivain à nous donner, dans une histoire générale du Canada, la liste des sources consultées. Parmi ces sources, les Véritables Motifs des Messieurs et Dames de la Société de Montréal (S.L. [ Paris ], 1643) sont mentionnés... Le jêsuitehistorien parle done de façon pertinente des fondateurs de Montréal, ceux demeurés en France, comme ceux habitant le Canada, tous besognant pour des fins missionnaires et civilisatrices, aidés souvent, de façon heureuse, par les pères de la Compagnie de Jésus.

68. - La Viel de la/ Vénérable Sœur/ Marguerite Bourgeois/, ditel du Saint-Sacrement/, institutrice, fondatrice, et première supérieure des filles/ séculières, de la/ Congrégation NotreDame,/ établie à Ville-Marie,/ dans/ l'isle de Montréal, en Canada./ Tirée des Mémoires certains, et la plupart/ originaux/. A Ville-Marie:/ Chez Wm. Gray, rue St. Paul/ 1818. 270 [1] pages. 
N.B. Cet ouvrage est attribué à Étienne Montgolfier, p.s.s., (17121791). "Dans l'Avis de l'Éditeur, celui-ci le désigne en remarquant que l'auteur anonyme du manuscrit qu'il livre à l'imprimeur, fut "chargé pendant [ de] longues années de gouverner la Congrégation [ de NotreDame ], et... qu'il en connaissait [ bien ] les traditions et les écrits... Son nom seul, ajoute-t-il, est presque une autorité dans la Province." Le manuscrit aurait done été composé avant l'année 1789, puisque M. Montgolfier, malade, cessait de lire et d'écrire à cette date. (Cf. Henri Gauthier, p.s.s. Sulpitiana, Montréal, 1926, p. 234-236). Un quart de siècle de manipulations par des lecteurs peut-être peu soucieux, ou ne comprenant pas la gravité d'interpoler les textes pour quelque raison que ce soit, explique bien des imprécisions et des erreurs dans cette œuvre. Toutefois, elle garde son importance à cause de son voisinage avec des documents de première main, qui existaient à cette date dans toute leur intégrité et qu'on désignait sous le nom de Écrits autographes de Sceur Bourgeoys [ vers 1698 ].

Nous y cueillons des souvenirs précieux, où apparaissent des Messieurs et Dames de Montréal que la bienheureuse Marguerite Bourgeoys connut fort bien: Pierre Chevrier, baron de Fancamp, l'abbé Pierre-Denis Le Prêtre et son frère Louis, seigneur de Fleury, M. de Bretonvilliers, 2e supérieur de Saint-Sulpice, M. de la Dauversière, le saint fondateur de la Société, M. de Maisonneuve, Jeanne Mance, MM. Louis et Charles d'Ailleboust, Madame Louis d'Ailleboust...

Il faut mentionner une liste des Messieurs et Dames de Montréal que la fondatrice de la Congrégation a dressée dans ses Ecrits autographes. Vingtdeux noms y sont alignés. Ce n'est pas la première nomenclature d'associés que nous connaissions. En 1672, dans son Histoire anonyme du Montréal, Dollier de Casson, p.s.s. nous présentait la première liste connue, où il entre 28 associés. Les noms, un peu estropiés, sont tout à fait reconnaissables. En outre, M. Dollier donne un chiffre total définitif: environ 45 associess. Il faut se rappeler ici que la Relation du Père Vimont, s.j., en 1642, mentionnait la présence à Notre-Dame de Paris, en février 1642, de 35 associés, en France. Il ne les nomme pas cependant. (Voir Histoire du Montréal, éd. Flenley, Toronto, 1928, p. 102-104). Sœur Morin, dans ses Annales de l'Hôtel-Dieu de Montréal, commencées en 1697, nous offre la $2 \Theta$ liste du même groupement avec 20 membres apparaissant au tableau. Les éditeurs de Sœur Morin, en 1921, ont rétabli en note les noms propres écrits tant bien que mal par Sœur Morin (Voir p. 42-43 des Annales, éditées par MM. Fauteux, Massicotte et Bertrand).

69. - François-Alexandre Aubert de La Chesnaye-Desbois, 1699-1789. Dictionnaire de la noblesse de France, 15 vol., in-4 (12 vol. plus 3 suppléments) Paris, 1784-1786.

N.B. C'est M. Badier qui réédita l'œuvre de cet auteur dont une réimpression fut publiée en 1875. L'édition originale porte les millésimes de 1757-1765, et comprenait 3 vol. et 4 suppléments de format in-8. Elle 
fut bientôt réimprimée en 12 vol. in-4, de 1770 à 1777 , puis de nouveau en 10 vol., in-4, à Paris, chez Schlesinger frères, en 1863-1867. On consulte encore La Chesnaye-Desbois, ne fût-ce que pour corroborer certains détails généalogiques, des dates, des noms diversement identifiés. Nous l'avons consulté au sujet des Séguier, de Madame Séguin, de Laisné de la Marguerie, d'autres encore. Nous ne l'avons pas regretté, au sujet de Madame Séguin, que l'abbé Verreau nous donnait à tort comme une Sanguin de Livry. M. Dollier de Casson avait parfaitement raison d'inclure cette janséniste, amie de la Chancelière Séguier, sous le nom de Séguin.

Rappel. - Voir aussi, sur le 18e siècle, les nos 7, 9, 11, 14, 48, $49,51,52,54,55,56$ de cette bibliographie (1e partie).

Note. - Nous ne pouvons citer actuellement, que fort peu d'ouvrages groupant les Messieurs de Montréal, au 18e siècle. D'autres historiens s'adonnant aux mêmes recherches sont ou seront peut-être plus heureux.

\section{C. - LE 19e SIÈCLE}

70. - Jacques Viger, 1787-1858. - [ Le 200e anniversaire de la fondation de Montréal ]. Article inséré dans les Mélanges religieux, Montréal, année 1842, p. 389 note.

N.B. L'érudit distingué de son époque, M. Viger, nous fournit dans une note de l'article ci-dessus mentionné, la quatrième liste connue des membres de la Société de Montréal. Il avait réussi à dépister 41 d'entre eux. Ajoutons qu'il venait de recevoir une aide puissante de France: la Vie de M. Olier, édition de 1841, en deux volumes, du savant sulpicien M. Faillon. Celui-ci inaugurait avec cette biographie toute une série de travaux originaux sur l'histoire de Montréal. Peu de documents lui échappèrent grâce à ses pérégrinations dans les cités des livres de son pays, d'ailleurs aussi, et dans les visites aux nombreux bureaux d'archives d'Europe, tout particulièrement.

Le Séminaire de Saint-Sulpice, à Paris, possédait alors, à lui seul, combien de pièces rarissimes lui venant de sa seigneurie de Montréal, depuis 1663. M. Viger connut très bien M. Faillon, lors de son passage à Montréal, en 1850, alors qu'il revenait du séminaire sulpicien de Baltimore, (E.U.).

71. - Augustin Jal, 1795-1873. - Dictionnaire critique de biographie et d'histoire. Deuxième édition, augm. Paris, Plon, 1872. IV-1357 pages. In-4.

N.B. Ouvrage de référence très utile à consulter. L'édition originale porte le millésime de 1867. Le dictionnaire de Jal est devenu rarissime, malheureusement. 
72. - Henry Harrisse, 1830-1910. - Notes pour servir à l'histoire la bibliographie et la cartographie de la NouvelleFrance (1545-1700). Paris, 1872. 376 pages. In-8.

N.B. Quel savant que M. Harrisse, ce familier du salon de George Sand (à qui il a dédie cet ouvrage), et de son groupe de littérateurs! On y trouve des notes copieuses de caractère historique tout autant que bibliographique à travers l'ouvrage.

73. - Émile Raunie, 1854-1911. Épitaphier du vieux Paris. 3 volumes. Paris, 1890-1901. Grand in-4. (Dans Histoire générale de Paris, 1866-1912, vol. 29-31).

N.B. Ancien élève de l'École des Chartes, licencié ès lettres, rédacteur au Ministère de l'Instruction publique, ce parfait érudit a écrit sur les ś́pultures célèbres de Paris de façon fort pertinente, définitive. Plusieurs des Messieurs et Dames de Montréal reposent sous des tombes fastueuses dans les Chapelles des monastères de la Capitale. Quelques-uns durent omigrer, tels les membres des familles Bullion et Lamoignon qui dormaient côte à côte dans une des chapelles latérales du couvent des Cordeliers à Paris. La pique des ouvriers, en creusant dans les ruines du monastère, pour y élever une autre construction, mit à jour leurs vieux cercueils (vers 1884). Pour leur part, ceux des Bullion (Claude, le surintendant, mort en 1640, et sa femme, Angélique Faure, "la bienfaitrice inconnue") réintégrèrent la chapelle de leur ancien Château de Wideville (Seine-et-Oise). On peut aujourd'hui y faire une visite, tout en admirant les splendides armoiries de la famille placées tout autour du petit sanctuaire.

74. - Abbé Hospice-Anthelme Verreau (1828-1901). Notice sur les fondateurs de Montréal. Montreal, Dawson Frères, Libraires, 1884. [ 12 pages ] 30 x $23.5 \mathrm{~cm}$.

N.B. Édition originale formée d'une étude parue dans les Mémoires de la Société Royale, section I, 1882, p. 95-106. Cette notice n'est au fond qu'une liste nouvelle (la cinquième) des Messieurs et Dames de Montréal avec quelques commentaires d'un grand érudit de chez nous, l'un des premiers présidents de la Société historique de Montréal, l'abbé Verreau. Quarante et un membres de la Société, fondatrice de notre ville forment cette galerie. On trouve cette $5 \Theta$ liste insérée également dans les Véritables Motifs... éd. de la Société historique de Montréal, présentée et annotée par l'abbe Verreau (Montreal, 1880?). Nous plaçons nous-même ce point d'interrogation à la suite du millésime, car l'abbé Verreau commençait, à la séance de la Société royale, le 25 mai 1882, la lecture de son étude sur les fondateurs de Montréal, par ces mots: "La Société historique de Mont- 
réal reproduit en ce moment (en 1882), dans la neuvième livraison de ses Mémoires, un petit livre d'une très grande rareté...: Les Véritables Motifs, etc. Il est facile de conclure. La $5 e$ liste des Associess de Montréal a donc өté, en 1882, imprimée deux fois, bien que les imprimeurs aient donné deux autres millésimes: pour les Véritables Motifs... 1880, au lieu de 1882; pour la Notice sur les fondateurs (ce tirage à part d'un Mémoire de la Société Royale) pour lequel on a composé une couverture, probablement en 1884) 1884, au lieu de 1882, date du mémoire lui-même.

75. - Adrien Launay, p.m.-e. [ de France]. - Histoire générale de la Société des Missions-étrangères. Pierre Téqui, librairie-éditeur, 82 rue Bonaparte, 1894. 33 volumes. 23.5 x $14.5 \mathrm{~cm}$.

N.B. Trois Messieurs de Montréal, et de la Compagnie du SaintSacrement, Antoine Barillon de Morangis, Christophe Duplessis-Montbard et Jean Garibal, baron de Saint-Sulpice, ont joue un rôle important dans la fondation de la Société intimement liée durant bien des années à la vie religieuse de la Nouvelle-France.

76. - Francis Parkman, 1823-1893. - The Jesuits in North America in the seventeenth century... Boston, Little, Brown, 1897. 2 volumes. In-8. (The Works, Champlain ed., vol. 3-4).

77. - Le Même. - The Old Regime in Canada. Boston, Little, Brown 1897. 2 volumes. In-8 (The Works, Champlain, ed., vol. 7-8.)

N.B. Des traductions existent de ces œuvres. Mais elles laissent un peu à désirer... Parkman, ce lettré artiste et cet historien, fut l'ami tout comme l'admirateur de l'abbé Casgrain, malgré de profondes divergences de vues religieuses. "Sans doute, a déclaré Aegidius Fauteux, lors de la célébration du centenaire de naissance de Parkman, à l'Université McGill, le 13 novembre 1923, à cause de préjugés inévitables, Parkman n'a pu voir toutes choses du même œil que nous... A certains moments, sa main peut se poser un peu trop lourdement sur ce qui nous tient au cœur comme catholiq ues..." L'on pense bien que certains Messieurs de Montréal, M. de La Dauversière en particulier, n'ont été ni compris, ni jugés très favorablement par cet Américain, sincèrement attaché à ses croyances protestantes.

78. - Dictionnaire de thøologie catholique... [ commence] sous la direction de A. Vacant... [ continuée sous celle de $\mathbf{M}$. Mangenot... puis M. Amann...] Paris, Letouzey et Ane, editeurs, 47, rue du Vieux-Colombier, 1899-1951. 15 volumes (150 fascicules.) $29 \times 21 \mathrm{~cm}$. 
N.B. On vient d'achever la publication de ce monument de la science religieuse catholique. J'ai devant moi la liste des collaborateurs du premier fascicule (1899). J'y relève 40 noms de savants dont 7 jésuites, 6 bénédictins. Les quarante théologiens sont tous professeurs dans les grandes universités et les séminaires réputés de toutes les parties du monde catholique. Nous y trouvons le nom d'un Canadien éminent: le Père Ephrem Longpré, o.f.m. Chaque article est signé. L'ordre alphabétique a été adopté naturellement et l'on trouvera les quelques Messieurs de Montréal auxquels on a consacré un article dispersé à travers les innombrables pages de l'ouvrage. MM. Olier, de Renty, de Ventadour et autres ne méritaientils pas une place de choix, parmi les doctes et saints personnages qu'on y voit paraître?

Rappel: voir aussi sur le $19 \Theta$ siècle, les nos $9,14,52,54,55,56$ de cette bibliographie (1e partie).

\section{LE 20e SIÈCLE}

79. - Université de Paris. La Compagnie du Très SaintSacrement de l'Autel. La "Cabale des dévots", 1627-1666. Thèse présentée à la Faculté de théologie protestante de l'Université de Paris, pour obtenir le grade de docteur en théologie et soutenue publiquement le 12 novembre 1902 , à 2 heures, par Raoul Allier. Paris, Librairie Armand Colin, 5. rue de Mézières, 1902.448 pages. $23 \times 15 \mathrm{~cm}$.

N.B. Nous avons préféré nous en tenir à la thèse elle-même, une édition assez rare aujourd'hui. Nous avons tout de même consulté l'édition ordinaire... M. Allier est devenu, depuis 1902, une autorité indispensable à consulter sur la Compagnie du Saint-Sacrement. Évidemment, nous demeurons mal à l'aise vis-à-vis de l'hostilité sourde ou très apparente que l'auteur manifeste sans cesse contre la Compagnie et contre des menées que tant d'historiens qualifient aujourd'hui d'intolérantes. Mais à côté de gestes malheureux, que nous devons juger pourtant selon les lumières de l'époque, que de bien accompli dans les domaines les plus divers! La Société de Notre-Dame de Montréal est considérée pour la première fois comme Comité de la Compagnie du Saint-Sacrement, dans l'ouvrage de M. Allier... Nous en avons dit quelques mots dans le No 59 de cette bibliographie. M. de la Dauversière et tous les associés, de la première à la dernière heure, dans l'œuvre missionnaire et colonisatrice de Montréal, y sont mentionnés dans un court récit inspiré des œuvres de M. Faillon. Disons toutefois que certaines circonstances rapportées par l'histoire n'y apparaissent point. Il y a de l'imprécision aussi pour certaines dates. Bref, tout est vraiment trop condensé autour des origines de Montréal. La 
part prépondérante de quelques-uns, la simple entr'aide accordée par d'autres, s'y trouvent mal établies, mal éclairées. "Pourquoi d'Argenson, conclut M. Allier (p. 150) a-t-il passé sous silence la part évidente de la Compagnie en toute cette histoire?" Sans doute, pourrions-nous répondre, c'est que Voyer d'Argenson n'avait vu mentionné nulle part dans les documents de la Compagnie du Saint-Sacrement, ce prétendu Comité de l'œuvre de Montréal, que M. Allier s'obstinait à y créer.

80. - Émile Salone, né en 1858. - La Colonisation de la Nouvelle-France. Études sur les origines de la nation canadiennefrançaise. Troisième édition. Paris, Librairie orientale et américaine. E. Guilmoto, éditeur, 6, rue de Mézières, [ 1908 ] XII 467 pages. Cartes, $23 \times 15 \mathrm{~cm}$.

N.B. Ouvrage d'une tenue classique, couronné par l'Académie française (prix Thérouanne, 1907). M. Salone, ancien élève de l'École des Chartes, docteur ès lettres, agrégé d'histoire et de philosophie, nous a certainement consacré une de ses meilleurs œuvres. Elle fait autorité chez nous. Je connais peu de pages sur la fondation de Montréal, démontrant une telle intelligence historique autour des documents et des vieux textes d'historiens; et, avec cela, présentées dans un style aussi précis que clair. Il faut lire et bien s'assimiler les pages allant de 73 à 82 . M. de la Dauversière a été vu dans son véritable rôle de fondateur de Montréal par M. Salone, alors que peu d'historiens l'admettaient encore avec une pareille autorité. Les associés de Montréal sont mentionnés, pour la plupart, dans cette dizaine de pages. Des notes et des références viennent assurer le lecteur de leur solidité.

81. - Abbé Henri Bremond, 1855-1933. - Histoire littéraire du sentiment religieux en France, depuis la fin des guerres de religion jusqu'à nos jours. Paris, Bloud et Gay, 3, rue Garancière, 1916-1936. 12 volumes. Portraits, figures, fac-similés. $25 \times 16.5 \mathrm{~cm}$.

N.B. Le volume 12 qui est posthume, présente un index alphabétique et analytique couvrant 256 pages. C'est l'œuvre de Charles Grolleau. Grâce à ce travail technique, qui ne compense pas pour l'absence de la bibliographie, nous n'avons pas à regretter une négligence absolue de l'appareil critique. Les notes et les références de l'abbé Brémond nous révélaient sans cesse au cours de l'œuvre, l'abondance et la richesse de son information. La citation des sources, s'ordonnant dans une bibliographie critique et analytique, aurait été et serait encore une aide puissante pour les historiens pénétrant dans les domaines du mysticisme français. Nous retraçons l'action de plusieurs Messieurs et dames de Montréal au cours de cet ouvrage d'envergure, écrite avec une science qui n'a d'égale que la finesse des jugements et le bon goût littéraire. 
82. - Les Anciens chateaux de France; notices historiques et descriptives par J. Vacquier, H. Soulange-Bodin [ et autres ]. Paris, Contet, 1920. [ 16 séries de gravures, in-folio, ont paru avant la guerre $1939-1945$ ].

N.B. Nous avons trouvé dans cette luxueuse collection de gravures décrivant l'intérieur et l'extérieur de somptueux châteaux français, des renseignements précieux sur Madame Claude de Bullion, et aussi sur Louis-Henry Habert de Montmor ou Montmort. MM. Vacquier et Soulange-Bodin, à la fois historiens et artistes, nous paraissent avoir tout lu sur le Grand Siècle. Leurs notices s'appuient sur de nombreuses références. C'est grâce à leurs travaux que nous apprenons où logent les archives des Bullion. Elles y étaient avant la dernière guerre, admirablement classées. Ce n'est plus aujourd'hui qu'un amas de vieux papiers qu'on a sauvés des bottes allemandes - et encore! - en les glissant en hâte, dans une confusion indescriptible, dans un coin de la vieille tour, tout près du château de Wideville. Il faudra des.mois et des mois pour s'y comprendre et en tirer profit. Et nous parlons de ce que nous avons vu, en compagnie du régisseur actuel, M. Dezouches, si parfaitement respectueux et attaché aux souvenirs de Wideville.

$$
\text { (à suivre) }
$$

